\title{
THE DESIGN OF WIRELESS SENSOR NETWORKS NODE FOR MEASURING THE GREENHOUSE'S ENVIRONMENT PARAMETERS
}

Cheng Wang, Chunjiang Zhao", Xiaojun Qiao, Xin Zhang, Yunhe Zhang National Engineering Research Center for Information Technology in Agriculture Shuguang Huayuan Middle Road 11\#, Beijing China, 100097

* Corresponding author, Address: P.O. Box 121, EU-China Center for Information \& Communication Technologies, China Agricultural University, 17 Tsinghua East Road, Beijing, 100097, P. R. China, Tel: +86-10-51503411, Fax: +86-10-51503705, Email: Zhaocj@nercita.org.cn

Abstract: Wireless sensor networks are integrated of sensor techniques, embedded system techniques, distributed computation techniques and wireless communication techniques. It's a hot research area in the world. It will be widely used in agriculture. This paper introduces the hardware and software design of wireless sensor networks node for the measurement of greenhouse environment parameters. These nodes can form networks themselves, measure the environment parameters precisely, transfer data safely, and solve the difficulty of wiring in greenhouse, making the earlier research of wireless sensor networks used in greenhouse

Keywords: wireless sensor networks, node, greenhouse, environment parameters, msp430

\section{INTRODUCTION}

As a new cropper plant technology, greenhouse is not limited by many factors such as plant zone, nature environment, climate and so on. It is important for agricultural production. Greenhouse environment parameters' measurement is a key tache of greenhouse's automation and efficiency. In

Wang, C., Zhao, C., Qiao, X., Zhang, X. and Zhang, Y., 2008, in IFIP International Federation for Information Processing, Volume 259; Computer and Computing Technologies in Agriculture, Vol. 2; Daoliang Li; (Boston: Springer), pp. 1037-1045. 
traditional sensor data collection system, a lot of cable has been used, it brings many difficulties into system installment and greenhouse production.

Because of a new research area and broad application prospect, wireless sensor networks have been paid a lot of attention by many scholars. It is essential for agricultural workers bring wireless sensor network into agriculture production.

We have designed and made the wireless sensor networks node for measuring temperature, humidity, dew point, using its general analog or digital port, it can also measure soil moisture, $\mathrm{CO}_{2}$ concentration, nourishing cream's PH, EC and cropper's physiological parameters and other environment parameters in greenhouse.

The paper will take the node used in greenhouse for example to state hardware and software design.

\section{THE HARDWARE OF THE WIRELESS SENSOR NETWORKS NODE}

The hardware of the wireless sensor networks node (Fig. 1)

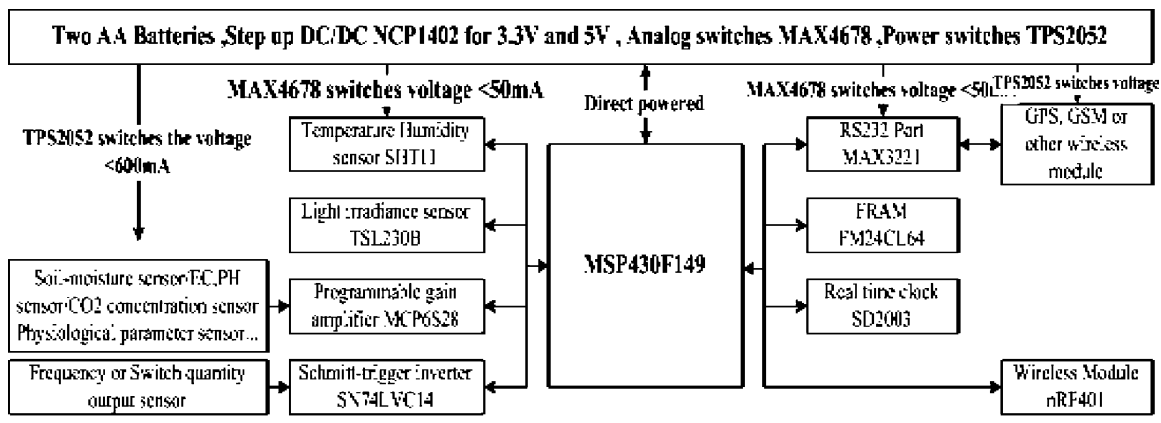

Fig. 1. Block diagram of the system hardware

\subsection{Performance of TI's MSP430F149}

The ultra low-power micro-controller $1 \mathrm{MSP} 430 \mathrm{~F} 149$ has $60 \mathrm{~KB}$ flash ROM and 2KB RAM. It has powerful processing ability (RSIC architecture, $125 \mathrm{~ns}$ instruction cycle time when using $8 \mathrm{M}$ crystal) and rich internal peripheral (12bit A/D converter, on-chip comparator, two USART/SPI ports, 48 I/O pins (Hu Dake, 2002) Ultra low-power is MSP430's most 
marked feature. It has low supply voltage range: $1.8-3.6 \mathrm{~V}$, five power-saving modes. When powered by $2.2 \mathrm{~V}, 1 \mathrm{MHz}$, the consume current is $280 \mathrm{uA}$. As it is powered by battery, node's energy is limited. This kind of microcontroller is the best choice for wireless sensor networks node, (Anton Muehlhofer, 2007, LutzBierI, 2002, E. Welsh, 2003).

\section{$2.2 \quad$ Sensors}

In this system the basic detected parameters are air temperature, air humidity, dew point, light irradiance.

Temperature, humidity and dew point are measured by SHT11 which is produced by Sensirion Company Switzerland. The SHT11 digital humidity and temperature sensor is fully calibrated, it offers long term stability and ease of use at very low cost. The digital CMOSens Technology integrates two sensors and readout circuitry on one single chip. Its measurement range is $0-100 \% \mathrm{RH}$, accuracy is $\pm 3 \% \mathrm{RH}$. Temperature range is $-40^{\circ} \mathrm{C}-+123.8^{\circ} \mathrm{C}$, and its accuracy is $\pm 0.4^{\circ} \mathrm{C}$ (Wang Hanzhi, 2004). Base on the data of temperature and humidity, we can work out the dew point precisely (accuracy is $\pm 1^{\circ} \mathrm{C}$ ).

Light irradiance is detected by TSL230B. This IC combines a configurable silicon photodiode and a current-to-frequency converter on single monolithic CMOS integrated circuits. The output can be either a pulse train or a square wave with frequency directly proportional to light intensity. The sensitivity of the device is selectable in three ranges, providing two decades of adjustment. The full-scale output frequency can be scaled by one of four preset values.

By timer's capture function, MSP430F149 can work out the value of frequency. Base on the output frequency and irradiance chart which is offered by the chip producer (Zou Wei, Kang Longyun, 2004), we can get the light irradiance (unit: $\mathrm{uW} / \mathrm{cm} 2$ ).

\subsection{General Analog and Digital Input Port}

For other parameters' detection, we designed the analog port to solve the standard $0-5 \mathrm{~V}, 1-10 \mathrm{~V}, 4-20 \mathrm{~mA}, 0-10 \mathrm{~mA}$ signals and even smaller signals which are exported by other sensors. It can be connected with eight kinds of different sensors. Standard current signals are transferred into voltage $(0-2.5 \mathrm{~V})$ signal by resistor, and standard voltage signal can be divided into the range of $0 \mathrm{~V}-2.5 \mathrm{~V}$. 
MCP6S28, the single-ended, rail-to-rail, programmable gain amplifiers (PGA), can be configured for 8 gains from $+1--32 \mathrm{~V} / \mathrm{V}$ and the input multiplexer can select one up to eight channels through an SPI port. [4] When does not want to amplify the signal, the PGA's gain is +1 as a follower to match the resistor between A/D convert and sensor.

The signal through the PGA and connects to MSP430F149's 12bit A/D converter.

Digital input port using six-Schmitt-trigger inverter SN74LVC14 to receive the data signal and filter the wave such as frequency, switch quantity, making the signal easy to receive by the micro-controller.

Users can choose different sensors to measure the different environment parameters in greenhouse.

Wireless Transfer Module:

Very low power consumption UHF transceiver chip nRF401 is designed to operate in the $433 \mathrm{MHz}$ ISM (Industrial, Scientific and medical) frequency band. It features Frequency Shift Keying (FSK) modulation and demodulation capability. It's transmit power can be adjusted to a maximum of $10 \mathrm{dBm}$. This system uses the differential antenna to increase communication length. This IC is connected to one of MSP430F149's USART port. In application the bit rate of this wireless transfer module is 20kbit/s, and communication length is over 300 meters (Fig. 2).

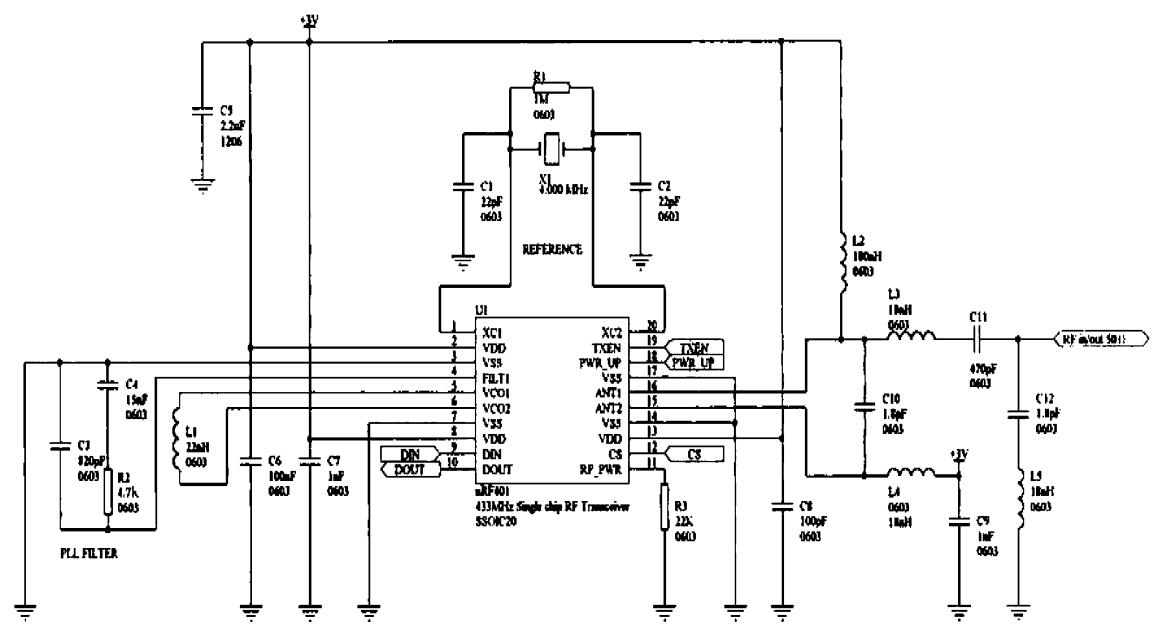

Fig. 2. nRF401 circuit diagram 


\subsection{Power Design and Management}

This system is powered by two AA batteries. Energy consumption is very important for system design. All systems take high efficiency, step-up DC/DC IC NCP1402 to booster the batteries' voltage up to 3.3V. The MAX4678 quad analog switches has $2 \mathrm{ohm}$ on-resistance. Each switch handles Rail-to-Rail analog signals and the current through it is $50 \mathrm{~mA}$. So MAX4678 has been used for switching the power of sensors, PGA and so on.

TPS2052 is also power switch for larger current.

When system is not collecting data, the MSP430 should turnoff the power of each sensor and switch into the sleep model to decreasing the power consumption. Main ICs' working current is show in Table 1.

Table 1. Working current of main ICs

\begin{tabular}{llll}
\hline ICs & & Active Model & Sleep Model \\
\hline MSP430F149 & $0.5 \mathrm{~mA}$ & $2.0 \mathrm{uA}$ \\
nRF401 & Receive & $0.28 \mathrm{~mA}$ & $8.0 \mathrm{uA}$ \\
& Transfer & $8.0 \mathrm{~mA}$ & - \\
SHT11 & $0.3 \mathrm{~mA}$ & - \\
FM24CL64 & $0.2 \mathrm{~mA}$ & - \\
SD2003 & $0.2 \mathrm{~mA}$ & $0.25 \mathrm{uA}$ \\
TSL230B & $1.2 \mathrm{~mA}$ & - \\
Other Sensors & $60 \mathrm{~mA}(\mathrm{Max})$ & - \\
Other consumption & $0.5 \mathrm{~mA}$ & $20 \mathrm{uA}$ \\
\hline
\end{tabular}

In case of each node works an hour on average, it consumes $5 \mathrm{~mA}$ (exclude other sensors). The system can work for over one year, so it is fit for greenhouse environment.

\section{SOFTWARE DESIGN}

\subsection{System Software}

It is composed of the main program, power management subroutine, different sensors measurement subroutine, nrf401 Rx/Tx subroutine.

The main program scheme (Fig. 3). The node is working in sleep model; other subroutine is called by interruption. In this operation, the system and networks' life can be prolonged. 


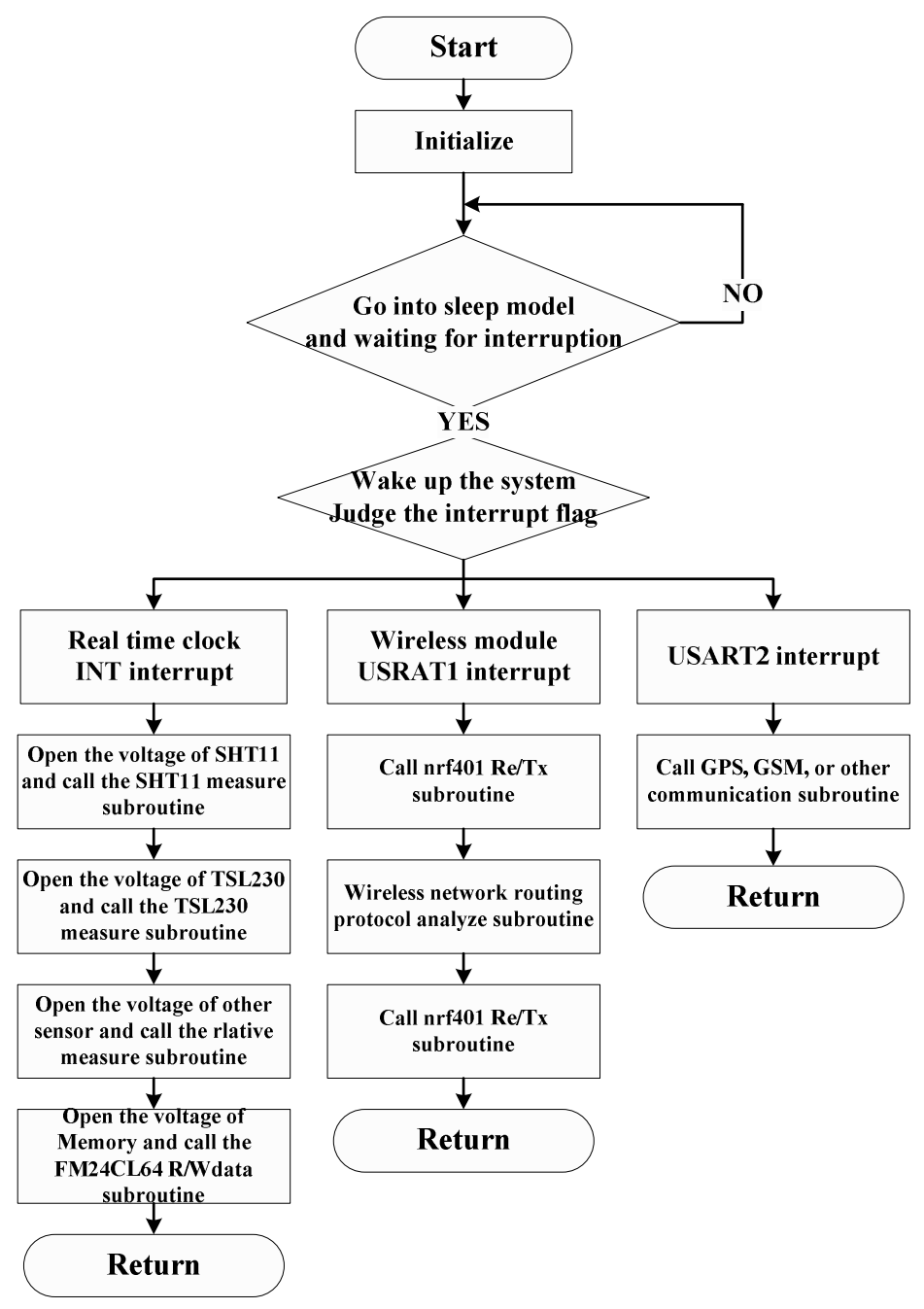

Fig. 3. Main program scheme

\subsection{Communication Protocol}

The key of the wireless data transmit subroutine. It is similar to the TCP/IP used in Internet. This protocol can achieve mesh, star or point to point networks (Akyildiz I.F., 2002).

Because of the limit of length, this paper only introduces simplify protocol. Its data packet format is designed as Table 2 shows. 
Table 2. Wireless transmission data packet format

\begin{tabular}{lllllllll}
\hline Lead1 & Lead2 & Mode & Length & MiddleID & LocalID & Destination & Data & Checksum \\
\hline 0xFF & 0xAA & 1Byte & 1Byte & 1Byte & 1Byte & 1Byte & nByte & 2Byte \\
\hline
\end{tabular}

In the test, OXff followed by 0xAA can restrain noise effectively. Mode is defined as data or instruction and master-slave transmit or retransmit mode. Length is the sum of MiddleID, LocalID, Destination and Data. Destination is the master node's address. LocalID is the local node's address. MiddleID is used when node is in retransmit mode. All this protocol keeps the data transfer safely. The exact network and communication protocol (Fig. 4) (Sohrabi K., 2000, L. Eschenauer, 2002, Warneke B., 2001).

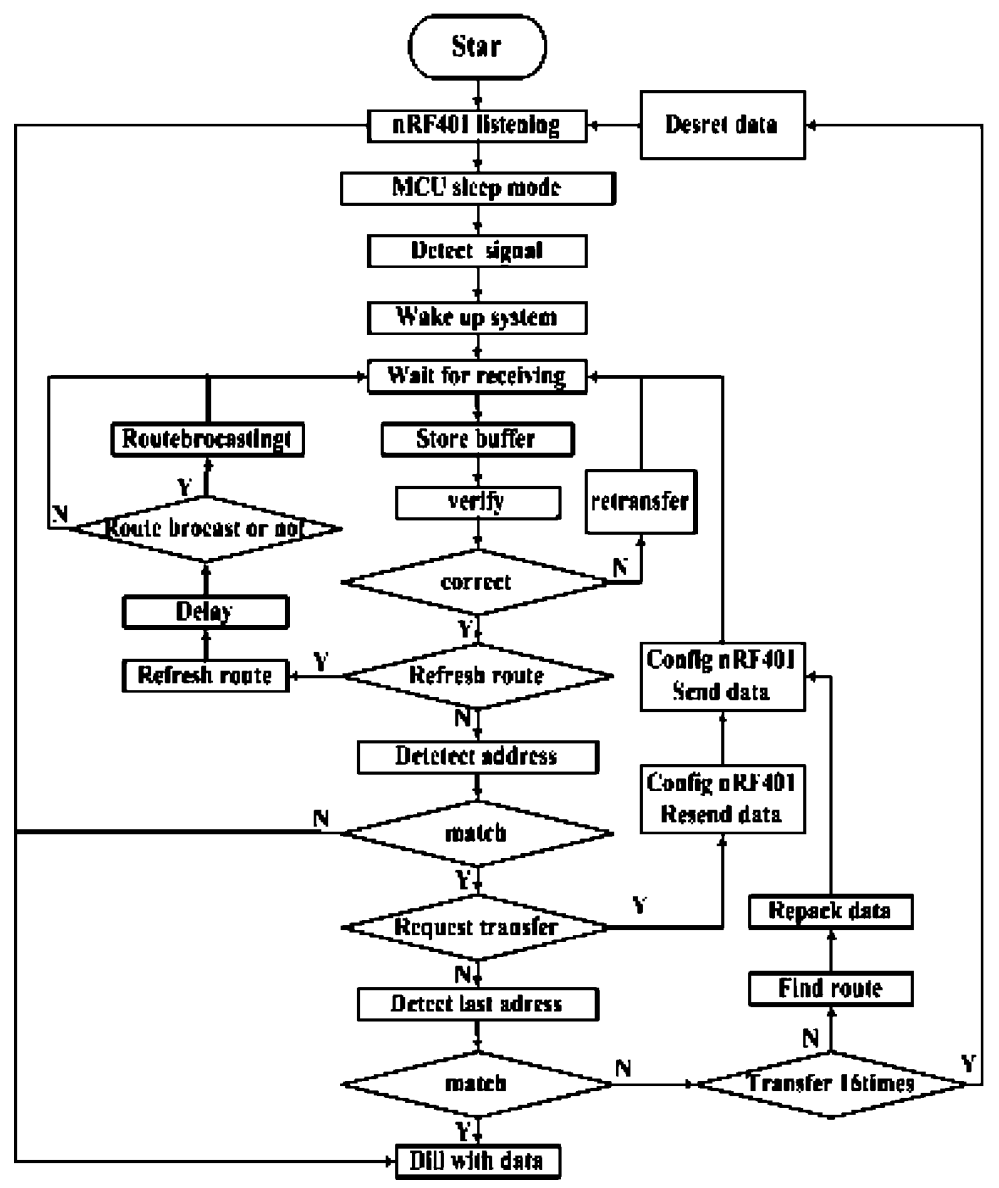

Fig. 4. Communication protocol 


\section{TEST AND RESULT}

In order to test the performance of nodes and networks and examine the ratio of the error code in transmitting, the test was conducted under the condition that a PC was used as the master and four nodes formed wireless sensor networks. Different parameters were collected and transmitted to the PC. There were no data lost or wrongly transmitted during the test. In addition, the distance of each node should be less than 300 meters, when in retransmit pattern the networks' area was not limited. Table 3 show the greenhouse's data collected by PC.

Table 3. Data collected in July 72007

\begin{tabular}{lcccc}
\hline Time & Temp & Humi & Light & CO2 \\
\hline 06:00:00 & 23.4 & -99.9 & 10.2 & 570 \\
06:30:00 & 24.1 & -99.9 & 19.3 & 586 \\
07:00:00 & 24.7 & -99.9 & 23.2 & 570 \\
08:00:00 & 25.3 & 99 & 26 & 578 \\
08:30:00 & 26.2 & 96 & 42.5 & 566 \\
09:00:00 & 27.8 & 91 & 50 & 542 \\
09:30:00 & 28.5 & 86 & 64.9 & 506 \\
$10: 00: 00$ & 30.8 & 80 & 84.8 & 498 \\
$10: 30: 00$ & 30.6 & 77 & 79.1 & 510 \\
$11: 00: 00$ & 30.5 & 76 & 63.1 & 510 \\
$11: 30: 00$ & 29.9 & 79 & 59.8 & 554 \\
$12: 00: 00$ & 30.1 & 75 & 66.1 & 546 \\
$12: 30: 00$ & 31.9 & 69 & 65.7 & 490 \\
$13: 00: 00$ & 33.2 & 65 & 80 & 526 \\
$13: 30: 00$ & 33.7 & 65 & 88.6 & 538 \\
$14: 00: 00$ & 32.4 & 67 & 96.8 & 510 \\
$14: 30: 00$ & 33.1 & 62 & 44.8 & 486 \\
$15: 00: 00$ & 30.3 & 72 & 30.9 & 522 \\
$15: 30: 00$ & 29.9 & 73 & 40.2 & 522 \\
$16: 00: 00$ & 30.4 & 72 & 32 & 510 \\
$16: 30: 00$ & 29.7 & 77 & 28.9 & 494 \\
$17: 00: 00$ & 29.6 & 75 & 25.7 & 502 \\
$17: 30: 00$ & 29.9 & 74 & 34.6 & 514 \\
& 29.4 & 75 & 22.3 & 530 \\
\hline
\end{tabular}

Battery charging test was showed (Fig. 5), Li+ battery was charged safe and fast.

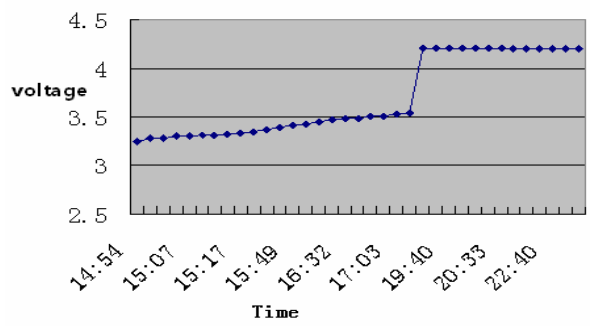

Fig. 5. Battery charge 


\section{CONCLUSION}

The design of wireless sensor networks node caters to the tendency of sensor's development: wireless and network. Bring the study of wireless sensor networks node into the measurement of greenhouse environment parameters, have solved a lot of difficulties in greenhouse. This node can not only be used in agriculture, but also in other fields, such as industry, academe, civil domain and so on (M. Srivastava, 2001).

\section{ACKNOWLEDGEMENTS}

This research is supported by the National High Technology Research and Development Program of China (863 Program, Grant 2006AA10A311 and Grant 2006AA10Z253) and Beijing Science \& Technology Program (Z0006321001391).

\section{REFERENCES}

$\mathrm{Hu}$ Dake. MSP430 series low power 16 bit micro-controller. Beijing University of Aeronautics and Astronautics Press, 2002 (3):5-10

Wang Hanzhi, Niu Zhenquan. Digital Humidity \& Temperature Sensor SHT11 and its Applications Based on the Technology of CMOSens®. Sensor World. 2004 (9):35-38

Zou Wei, Kang Longyun. The Device based on MSP430 Microcontroller for Wind Speed and Light Irradiance Testing Synchronously. Information of Microcomputer, 2003 (19)11:56-58

Xian Lijuan, Li Shengyu, Yang Shizhong. The application of programmable gain amplifier MCP6S2X in signal collection. International Electronic Elements, 2004 (2):71-73

Akyildiz I.F., Su Weilian, Cayirci E.A survey on sensor networks. IEEE Communications Magazine, 2002 (8):102-114

Sohrabi K., Gao J., Ailawadhi V., et al. Protocol for self-organization of a wireless sensor network. IEEE Personal Communication, 2000 7(5):16-27

L. Eschenauer and V.D. Gligor. A key-management scheme for distributed sensor networks, In Proceedings of the 9th ACM Conference on Computer and Communication Security, 2002 (22):41-47

Warneke B., Last M., Liebowitz B., et al. Smart dust: communicating with a cubicmillimeter computer. IEEE Computer, 2001 34(1):44-51

Anton Muehlhofer Controlling the DCO Frequency of the MSP430x11x. Texas Instrument Deutschland Application Report SLAA074 2007

LutzBierI. Interfacing the 3V MSP430 to 5V Circuits. Application Report SLAA148 2002.10 
M. Srivastava, R. Muntz, and M. Potkonjak, Smart Kindergarten: Sensor-based Wireless Networks for Smart Developmental Problem-solving Environments, Intl. Conf. on Mobile Computing and Networking 2001, Rome, Italy, July 2001, pp. 132-138

Rice's GNOMES: E. Welsh, W. Fish, P. Frantz, GNOMES: A Testbed for Low-Power Heterogeneous Wireless Sensor Networks, IEEE International Symposium on Circuits and Systems (ISCAS), Bangkok, Thailand, 2003 (5): 15-20 